\title{
Inhibition of UCHL1 by LDN-57444 attenuates Ang II-Induced atrial fibrillation in mice
}

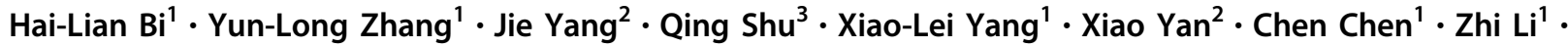 \\ Hui-Hua $\mathrm{Li}^{1}$
}

Received: 27 April 2019 / Revised: 23 September 2019 / Accepted: 1 October 2019 / Published online: 7 November 2019

(c) The Author(s) 2019. This article is published with open access

\begin{abstract}
Atrial fibrillation (AF) is the most common human arrhythmia in clinical practice and may be promoted by atrial inflammation and fibrosis. Ubiquitination is an important posttranslational modification process that is reversed by deubiquitinating enzymes (DUBs). DUBs play critical roles in modulating the degradation, activity, trafficking, and recycling of substrates. However, less research has focused on the role of DUBs in AF. Here, we investigated the effect of ubiquitin C-terminal hydrolase 1 (UCHL1), an important DUB, on the development of AF induced by angiotensin II (Ang II). Male wild-type mice were treated with the UCHL1 inhibitor LDN57444 (LDN) at a dose of $40 \mu \mathrm{g} / \mathrm{kg}$ and infused with Ang II ( $2000 \mathrm{ng} / \mathrm{kg} / \mathrm{min}$ ) for 3 weeks. Our results showed that Ang II-infused wild-type (WT) mice had higher systolic blood pressure and an increased incidence and duration of AF. Conversely, this effect was attenuated in LDN-treated mice. Moreover, the administration of LDN significantly reduced Ang II-induced left atrial dilation, fibrosis, inflammatory cell infiltration, and reactive oxygen species (ROS) production. Mechanistically, LDN treatment inhibited the activation of multiple signaling pathways (the AKT, ERK1/2, HIF- $1 \alpha$, and TGF- $\beta /$ smad2/3 pathways) and the expression of CX43 protein in atrial tissues compared with that in vehicle-treated control mice. Overall, our study identified UCHL1 as a novel regulator that contributes to Ang II-induced AF and suggests that the administration of LDN may represent a potential therapeutic approach for treating hypertensive AF.
\end{abstract}

Keywords Angiotensin II $\cdot$ Atrial fibrillation $\cdot$ Deubiquitinase $\cdot$ Fibrosis $\cdot$ UCHL1

Supplementary information The online version of this article (https:// doi.org/10.1038/s41440-019-0354-z) contains supplementary material, which is available to authorized users.

Hai-Lian Bi

diana_521@163.com

$\triangle$ Hui-Hua Li

hhli1935@aliyun.com

1 Department of Cardiology, Institute of Cardiovascular Diseases, First Affiliated Hospital of Dalian Medical University, Dalian 116000, China

2 Department of Nutrition and Food Hygiene, School of Public Health, Dalian Medical University, Dalian 116044, China

3 Affiliated Zhongshan Hospital of Dalian University, Dalian University, Dalian 116000, China

\section{Introduction}

Atrial fibrillation (AF), the most common clinical arrhythmia worldwide, has been well characterized, and it is associated with serious cardiovascular diseases and an increased risk of stroke and death. Although the pathophysiological mechanism of AF is not fully understood, atrial electrical and structural remodeling are the main factors of the persistence and progression of $\mathrm{AF}[1,2]$. Atrial fibrosis is the hallmark of atrial structural remodeling, which is a common feature of clinical AF [3]. Increasing evidence has demonstrated that the renin-angiotensin system (RAS) plays a key role in the initiation and development of AF. AngII (angiotensin II) exerts its biological functions via the activation of angiotensin type 1 receptor (AT1R) [4]. The activation of AT1R stimulates several downstream mediators, including AKT/ERK, TGF- $\beta /$ Smad, MAP kinases, NADPH oxidase, NF-kB, and hypoxia inducible factor-1alpha (HIF-1 $\alpha$ ), which are involved in vasoconstriction, fibrosis, inflammation, oxidative stress, 
and ion channel abnormalities [1, 5-7], Ang II-mediated inflammation and oxidative stress are thought to promote atrial fibrosis in $\mathrm{AF}[2,8,9]$. Thus, the inhibition of the AT1R-mediated activation of signaling is critical for blocking AF development.

Ubiquitination is a dynamic posttranslational modification that is naturally mediated by ubiquitin-activating (E1), ubiquitin-conjugating (E2), and ubiquitin ligase (E3) enzymes. This modification can be reversed by deubiquitinases (DUBs, also called deubiquitinating enzymes), which can regulate the degradation, trafficking, and activity of substrates [10]. Ubiquitin C-terminal hydrolase 1 (UCHL1), also called protein gene product 9.5 (PGP9.5), is an important member of the ubiquitin carboxy-terminal hydrolase (UCH) family of DUBs and catalyzes the hydrolysis of C-terminal ubiquityl esters and amides. Increasing data has indicated that UCHL1 plays an important role in proliferation, neoplasm metastasis, oxidative stress and immune responses, and it has been implicated in the development of neurodegenerative diseases and cancer [11-14]. A study suggested that UCHL1 influences skeletal muscle development and function. The deletion of UCHL1 in mice results in abnormal shuffling movements, hind-limb paralysis, and early death [15]. Recently, UCHL1 expression was shown to be highly upregulated in cardiomyocytes after myocardial infarction and to be associated with increased ubiquitin expression [16]. However, little is known about the role of UCHL1 in regulating AF and atrial remodeling.

In this study, we examined the effect of UCHL1 inhibition by LDN, a specific inhibitor, on the development of $\mathrm{AF}$ in a murine model of Ang II-induced AF. Our results showed that Ang II infusion significantly upregulated UCHL1 expression at both the mRNA and protein levels in the atria. The administration of LDN to mice reduced the Ang II-induced elevation of blood pressure, the inducibility and duration of AF, left atrial dilation, fibrosis, inflammation, and oxidative stress. This protective effect was associated with the inhibition of multiple signaling pathways (the AKT, ERK1/2, HIF- $1 \alpha$, TGF- $\beta /$ Smad $2 / 3$, and CX43 pathways). Thus, these results suggest that the inhibition of UCHL1 attenuates Ang II-induced AF. The UCHL1 inhibitor LDN may be a potential therapeutic drug for the treatment of hypertensive AF.

\section{Materials and methods}

\section{Animals and treatment}

Eight-week-old male C57BL/6 mice (the Jackson Laboratory, Bar Harbor, ME, USA) were infused with saline or Ang II (2000 ng/kg/min) (A107852; Aladdin, Shanghai,
CHN) using osmotic mini-pumps (Alzet model 1004; Durect, Cupertino, CA, USA) for 3 weeks as described previously [17]. The UCHL1 inhibitor LDN-57444 (40 $\mu \mathrm{g} /$ $\mathrm{kg}$ ) (MCE, Burlington, NJ, USA) or vehicle (DMSO) (Sigma-Aldrich, St. Louis, MO, USA) was administered intraperitoneally one time per day for 3 weeks beginning one day before Ang II infusion. This entire study was approved by the Animal Care and Use Committee of Dalian Medical University and conformed to the Guide for the Care and Use of Laboratory Animals published by the U.S. National Institutes of Health (NIH Publication No. 85-23, revised 1996).

\section{Blood pressure measurement}

The blood pressure of the mice was measured one day before LDN was administered and monitored every other day after Ang II infusion with a tail-cuff system (BP-2010, Softron, Tokyo, JPN), as described previously [18, 19].

\section{Arrhythmia inducibility and duration}

After 3 weeks of treatment, the mice were anesthetized by an intraperitoneal injection of $2.5 \%$ tribromoethanol ( 0.02 $\mathrm{mL} / \mathrm{g}$; Sigma-Aldrich, St. Louis, MO, USA) [20]. At the end of Ang II infusion, intracardiac pacing was performed in mice through the insertion of an eight-electrode catheter $(1.1 \mathrm{~F}$, octapolar EP catheter, Scisense, London, Ontario, Canada) via the jugular vein and its advancement into the right atrium and ventricle [20]. The inducibility of AF was measured by applying a 5 -s burst using an automated stimulator as described previously and recorded with a computer-based data acquisition system (GY6328B; Henan Huanan Medical Science and Technology, Co., Ltd., Zhengzhou, HA, CHN) [4, 20]. A series of bursts were repeated three times after stabilization for $5 \mathrm{~min}$. The duration of AF was defined as the time interval between a rapid and irregular atrial rhythm following the onset of $\mathrm{AF}$ and the onset of the first normal sinus beat.

\section{Histopathologic examinations}

The hearts were removed and fixed in $4 \%$ paraformaldehyde (Solarbio, Beijing, CHN) for $24-48 \mathrm{~h}$ and then embedded in paraffin. The samples were sectioned into 5$\mu \mathrm{m}$ sections and stained with Masson's trichrome (SigmaAldrich, St Louis, MO, USA) following the manufacturer's instructions as previously described [18]. Immunohistochemical staining was performed with anti-UCHL1, $\alpha$-SMA ( $\alpha$-smooth muscle actin) (ab7817; Abcam, Bristol, UK), F4/ 80 (ab6640; Abcam, Bristol, UK) antibodies [18]. Cryosections were prepared and stained with dihydroethidine 
(DHE; Sigma-Aldrich, St. Louis, MO, USA) as described [18]. Images of ten random fields per sample were taken at $\times 100$ or $\times 200$ magnification (Nikon, Tokyo, JPN) and were analyzed by ImageJ (NIH, Bethesda, MD, USA).

\section{Quantitative real-time PCR (qPCR) analysis}

Total RNA was isolated from LA tissues with TRIzol (Invitrogen) and reverse transcribed using a reverse transcription kit (RR047A; Takara, Tokyo, JPN) to synthesize first-strand cDNA [18]. qPCR amplification was performed using an iCycler IQ system (Bio-Rad, Plano, TX, USA). Primers used in the qPCR analysis are shown in Supplementary Table 1 (Sangon Biotech, Shanghai, CHN). The relative mRNA levels were normalized to those of the endogenous control (GAPDH).

\section{Western blot analysis}

Snap-frozen left atrial tissues were prepared and subjected to sonication in ice-cold RIPA buffer containing inhibitors and PMSF (100 mM; Solarbio, Beijing, CHN). Proteins were separated by electrophoresis on SDS-PAGE gels and blotted onto PVDF membranes (Millipore, Billerica, MA, USA). The appropriate antibodies, including UCHL1 (14730-1-AP; Proteintech, Wuhan, HB, CHN), AKT (\#9272; Cell Signaling Technology, Danvers, MA, USA), p-AKT (Ser473) (\#4060; Cell Signaling Technology, Danvers, MA, USA), ERK1/2(\#9102; Cell Signaling Technology, Danvers, MA, USA), p-ERK1/2 (Thr202/Tyr204) (\#9101; Cell Signaling Technology, Danvers, MA, USA), TGF- $\beta$ (\#3711; Cell Signaling Technology, Danvers, MA, USA), Smad2 (\#5339; Cell Signaling Technology, Danvers, MA, USA), p-Smad2/3(\#8828; Cell Signaling Technology, Danvers, MA, USA), CX43 (ab11370; Abcam, Bristol, UK) and $\alpha$-tubulin (11224-1-AP; Proteintech, Wuhan, HB, $\mathrm{CHN}$ ) antibodies, were used [18]. The blots were developed by using the enhanced chemiluminescent system (ECL Plus, Thermo Fisher, Waltham, MA, USA), and signal acquisition was performed using the FluorChem M system (ProteinSimple, San Jose, CA, USA). The intensities of the proteins were measured by ImageJ software and were normalized to the intensity of endogenous tubulin.

\section{Statistical analysis}

Statistical calculations were performed using SPSS 16.0 (SPSS, Chicago, IL, USA). All results are expressed as the mean \pm standard error of the mean (SEM). First, a normality test was performed. If the data were normally distributed, Student's $t$ test was used to test the difference between the two groups. If the data were not normally distributed, the Mann-Whitney $U$ test was used to test the difference between the two groups. $P<0.05$ was considered statistically significant.

\section{Results}

\section{Ang II infusion upregulates UCHL1 expression in the Atria}

To investigate whether UCHL1 activity is involved in regulating AF, we first tested the expression of UCHL1 in atrial tissues. The mRNA and protein levels of UCHL1 were significantly upregulated in Ang II-infused atrial tissues after 3 weeks of infusion $(2000 \mathrm{ng} / \mathrm{kg} / \mathrm{min})$ compared with saline-infused tissues (Fig. 1a, b). Furthermore, the increase in UCHL1 expression in Ang II-infused atrial tissues was confirmed by immunohistochemical staining (Fig. 1c). These data suggest that UCHL1 may play a role in Ang II-induced AF.

\section{Administration of LDN inhibits Ang II-induced systolic blood pressure and left atrial (LA) dilation}

We next determined whether the inhibition of UCHL1 activity regulates systolic blood pressure (SBP) and atrial structure remodeling. We established an AF mouse model through the infusion of a high dose of Ang II and administered the UCHL1 inhibitor LDN. After 3 weeks of Ang II

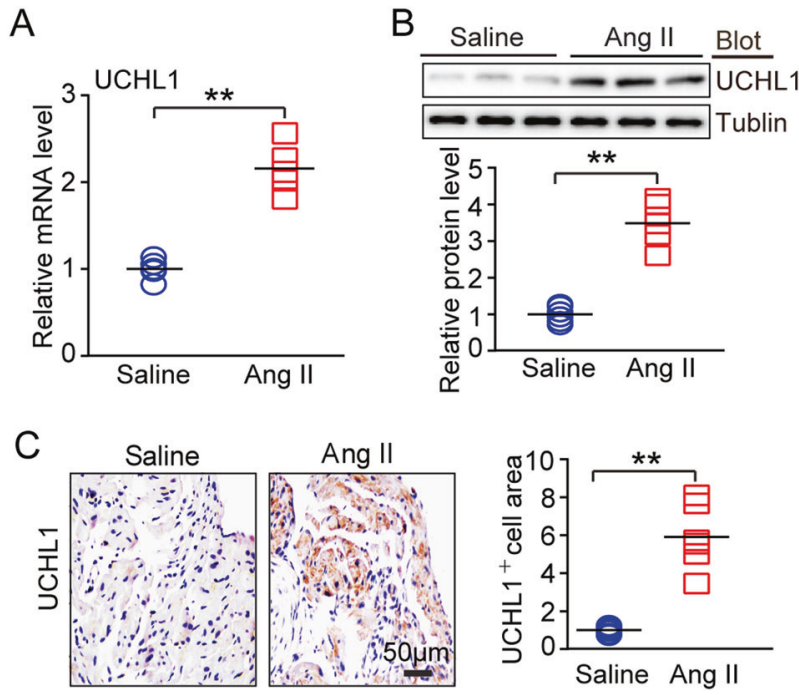

Fig. 1 Ang II infusion upregulates UCHL1 expression in the hearts. a Wild-type (WT) mice were infused with saline or Ang II (2000 ng/kg/ min) for 3 weeks. UCHL1 mRNA expression in the atrial tissues was detected by qPCR analysis. b UCHL1 protein levels in atrial tissues were detected by immunoblotting analysis (upper) and quantified (lower). c Atrial sections were stained with an anti-UCHL1 antibody by immunohistochemistry (left). Scale bar $=50 \mu \mathrm{m}$. The quantification of the UCHL1-positive area (right). ${ }^{*} P<0.05,{ }^{* *} P<0.01$ vs salinetreated WT mice 
treatment, the SBP was elevated in the vehicle-treated mice compared with the saline-treated mice. However, this effect was significantly reduced in the LDN-treated mice (Fig. 2a). Since atrial structural remodeling is a major risk factor for $\mathrm{AF}$ [4], we then examined the effect of LDN on atrial dilation in vivo. Echocardiography revealed that the Ang IIinduced dilation of the left atrium (LA) observed in vehicletreated WT mice was also attenuated in LDN-treated mice (Fig. 2b).

\section{Administration of LDN Attenuates Ang II-induced AF}

To determine the role of LDN in regulating AF development, the inducibility and duration of AF were examined in Ang II-infused mice treated with or without LDN. The inducibility of AF was appreciably increased in the Ang II-treated mice compared with the saline-treated mice (77.8\% versus $25.0 \%)$. Although LDN treatment reduced Ang II-triggered AF inducibility (57.1\% versus $77.8 \%$ ), no significant difference was observed between the LDNtreated mice and the vehicle (DMSO)-treated mice (Fig. 3a, b). Interestingly, the total duration of AF was substantially reduced in the LDN-treated mice compared with the vehicle-treated mice after Ang II infusion (Fig. 3c). There was no significant difference between the two groups treated with saline (Figs. 3a through c). The heart rate was not significantly changed between the vehicle-treated and LDN-treated mice after saline or Ang II infusion.

\section{Administration of LDN attenuates Ang II-induced fibrosis}

We then examined the role of LDN on atrial fibrosis, a hallmark of atrial remodeling. Ang II treatment, compared with saline treatment, significantly increased the atrial fibrotic area, whereas LDN treatment remarkably attenuated this effect (Fig. 4a). Moreover, the increase in the number of $\alpha$-SMA-positive myofibroblasts induced by Ang II infusion observed in the vehicle (DMSO)-treated WT mice was markedly attenuated in the LDN-treated mice (Fig. 4b). In addition, the increased mRNA expression of fibrotic markers (collagen I and III) induced by Ang II was also reduced in the LDN-treated mice (Fig. 4c, d). Together, these data suggest that the administration of LDN inhibits Ang IIinduced hypertension, left atrial dilation and fibrosis.

\section{Administration of LDN attenuates Ang II-induced inflammation and oxidative stress}

It has been reported that inflammation and oxidative stress play a critical role in regulating atrial fibrosis [21]. We further assessed whether LDN influences the inflammatory response and reactive oxygen species (ROS) production in the atria. Ang II infusion resulted in a marked increase in inflammatory cell infiltration, including that of F4/80-positive macrophages, in the vehicle-treated mice, but this increase was attenuated in the LDN-treated mice (Fig. 5a). Moreover, DHE staining revealed that the Ang II-induced
Fig. 2 Administration of LDN reduces pressure blood pressure elevation and improves atrial dysfunction after Ang II infusion. Male wild-type (WT) mice were infused with Ang II and injected intraperitoneally with LDN for 3 weeks. a Systolic blood pressure (SBP) was measured by a mouse tailcuff system before and after Ang II infusion. b Representative echocardiographs of LA dilation in wild-type mice treated with LDN or DMSO (control) after 3 weeks of saline or Ang II infusion. ${ }^{*} P<0.05,{ }^{* *} P<0.01$ versus saline control; ${ }^{\#} P<0.05$ versus Ang II alone
A

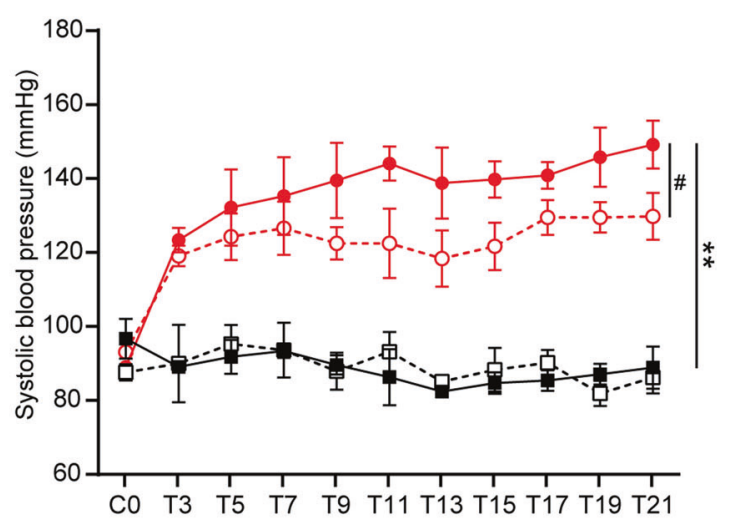

$\rightarrow-$ DMSO+Saline

- $\square$-. LDN+Saline

$\rightarrow$ DMSO+Ang II

- - -. LDN+Ang II

B

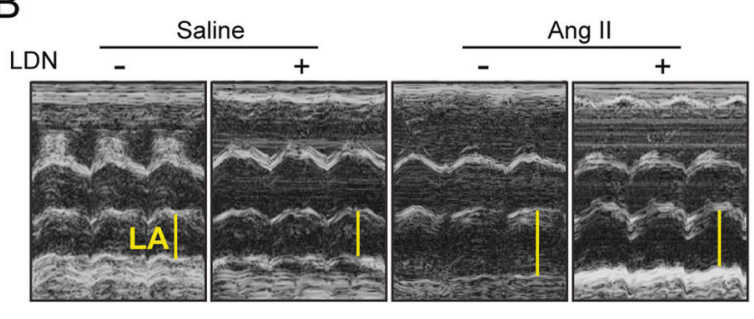


Fig. 3 Administration of LDN attenuates Ang II-induced AF. a Representative atrial electrogram recordings of DMSO-treated and LDN-treated mice infused with saline or Ang II for $21 \mathrm{~d}$. Burst pacing is indicated by the solid lines, and the dashed lines indicates AF. b The percentage of mice in which AF was successfully achieved in each group (bottom left; $n=7-9$ mice per group). c Total AF duration for each DMSO-treated and LDN-treated mouse infused with saline or Ang II, as determined by burst pacing
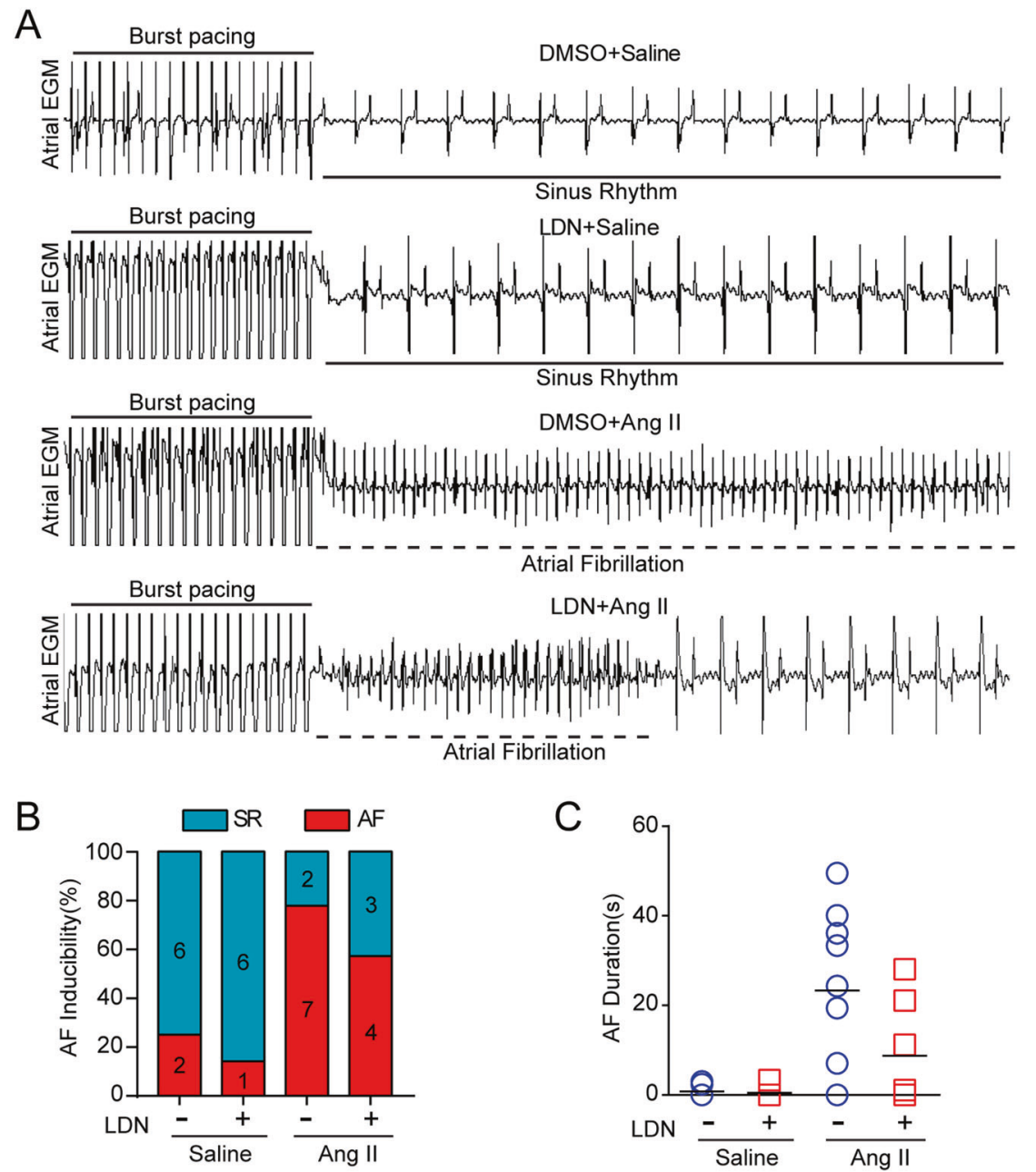

increase in superoxide production observed in WT mice was decreased in LDN-treated mice (Fig. 5b). Accordingly, the mRNA levels of IL-1 $\beta$ and IL-6 (proinflammatory cytokines) and NOX2 and NOX4 (NADPH oxidase subunits) were lower in atrial tissue from the LDN-treated mice than in tissue from the DMSO-treated mice with Ang II infusion (Fig. 5c-f). There was no significant difference in these parameters between the two groups treated with saline (Fig. 5a-f).

\section{Administration of LDN reduces multiple signaling pathways}

Ang II triggers multiple signaling pathways, such as AKT/ mTOR, HIF-1 $\alpha$, TGF- $\beta / \operatorname{Smad} 2 / 3$, and NADPH oxidase, which are involved in inflammation and fibrosis formation $[20,22]$. To study the molecular mechanism by which LDN protects against Ang II-induced AF, we investigated several signaling pathways associated with atrial fibrosis and inflammation. The administration of LDN, compared with vehicle, attenuated Ang II-induced increases in p-AKT, p-
ERK, HIF-1 $\alpha$, TGF- $\beta 1$, and p-Smad2/3 in the atria (Fig. 6a, b). In addition, the expression of CX43 was markedly attenuated in the LDN-treated mice compared with the vehicle (DMSO)-treated mice after Ang II treatment (Fig. 6c).

\section{Discussion}

This study is the first to confirm that UCHL1 expression is significantly upregulated in Ang II-infused atrial tissues. The inhibition of UCHL1 by LDN (a specific inhibitor) significantly reduced the Ang II-induced increase in blood pressure, atrial fibrillation, fibrosis, inflammation, and oxidative stress. These beneficial actions were possibly associated with the inactivation of multiple signaling mediators (AKT, ERK1/2, HIF- $1 \alpha$, and TGF- $\beta / \mathrm{Smad} 2 / 3$ ) in atrial tissues (Fig. 6). The working model is shown in Fig. 6c. In summary, our results indicate that UCHL1 plays an important role in Ang II-induced AF and may be a novel target for the treatment of hypertensive AF. 
A
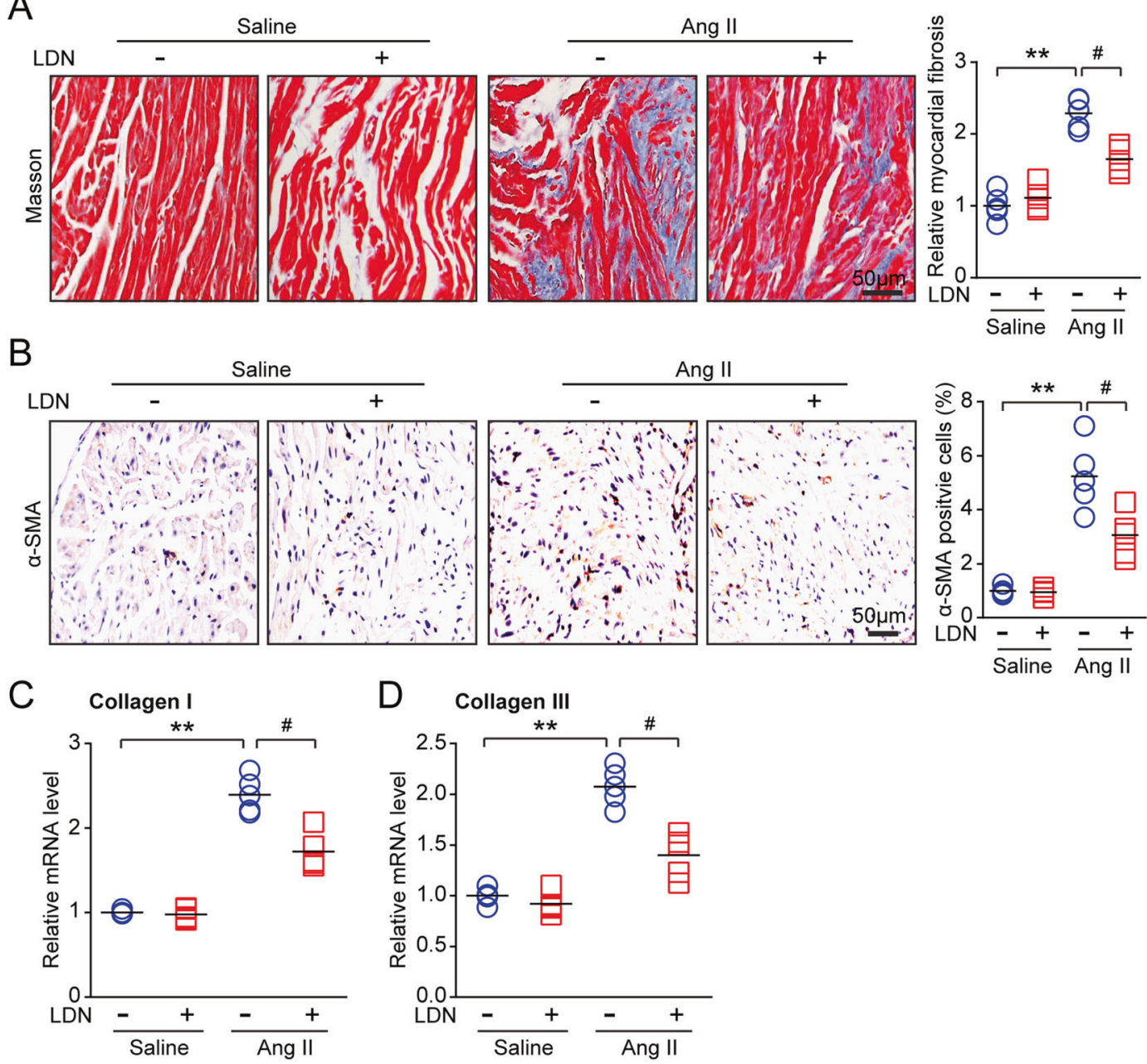

Fig. 4 Treatment with LDN suppresses Ang II-induced atrial fibrosis. Male wild-type (WT) mice were infused with Ang II and injected intraperitoneally with $\mathrm{LDN}(40 \mu \mathrm{g} / \mathrm{kg}$, one time per day) for $21 \mathrm{~d}$. a Representative images of Masson trichrome staining for atrial fibrosis (left). The quantification of the fibrotic area (right; $n=5$ mice per group). b Representative images of $\alpha$-SMA (a fibrosis marker)

immunohistochemistry (left). Then quantification of $\alpha$-SMA-positive cells (right; $n=5$ mice per group). $\mathbf{c}, \mathbf{d}$ qPCR analyses of collagen I and collagen III levels in atrial tissues. $n$ represents the number of animals. ${ }^{*} P<0.05,{ }^{* *} P<0.01$ versus saline control; ${ }^{\#} P<0.05$ versus Ang II alone

UCHL1 was originally identified as a neuron-specific protein highly expressed in the nervous system, and its mutations are associated with neurodegenerative diseases, including Parkinson's disease and Alzheimer's disease $[11,12]$. Previous studies have shown that UCHL1 is expressed at lower levels in healthy tissues, but it is highly induced in several forms of cancer and lung tumor cell lines [23]. UCHL1 is also upregulated in denervated skeletal muscles and pulmonary artery constriction (PAC)-treated right ventricular and infarcted heart tissues [16, 24, 25]. Moreover, UCHL1 has been implicated in regulating apoptosis of $\beta$ cells and diabetes progression in hIAPP-Tg mice $[26,27]$. In this study, our results showed that UCHL1 expression was also increased in the atria after Ang II infusion (Fig. 1), indicating that the upregulation of UCHL1 expression may influence AF susceptibility. Indeed, we demonstrated for the first time that the inhibition of UCHL1 activity by the inhibitor LDN attenuates Ang II-induced AF and atrial remodeling.

Ang II is involved in regulating various cardiovascular diseases, such as hypertension, cardiac hypertrophic remodeling, heart failure, and AF [28]. The activation of AT1R by Ang II triggers multiple signaling pathways, including the PI3K/AKT, MAPKs, NADPH oxidase, and TGF- $\beta$ / Smad pathways, which stimulate atrial fibrosis, inflammation, and oxidative stress, leading to $\mathrm{AF}$ inducibility $[4,20]$. Both the TGF- $\beta /$ Smad and Hif- $1 \alpha$ pathways have been demonstrated to be key signaling pathways in Ang IIinduced myocardial fibrosis [1]. HIF consists of an $\alpha$ subunit and a $\beta$-subunit. HIF- $1 \alpha$ is a critical transcription factor that is closely related to hypoxia, and increased NOX expression results in ROS production [29]. TGF- $\beta 1$ also 

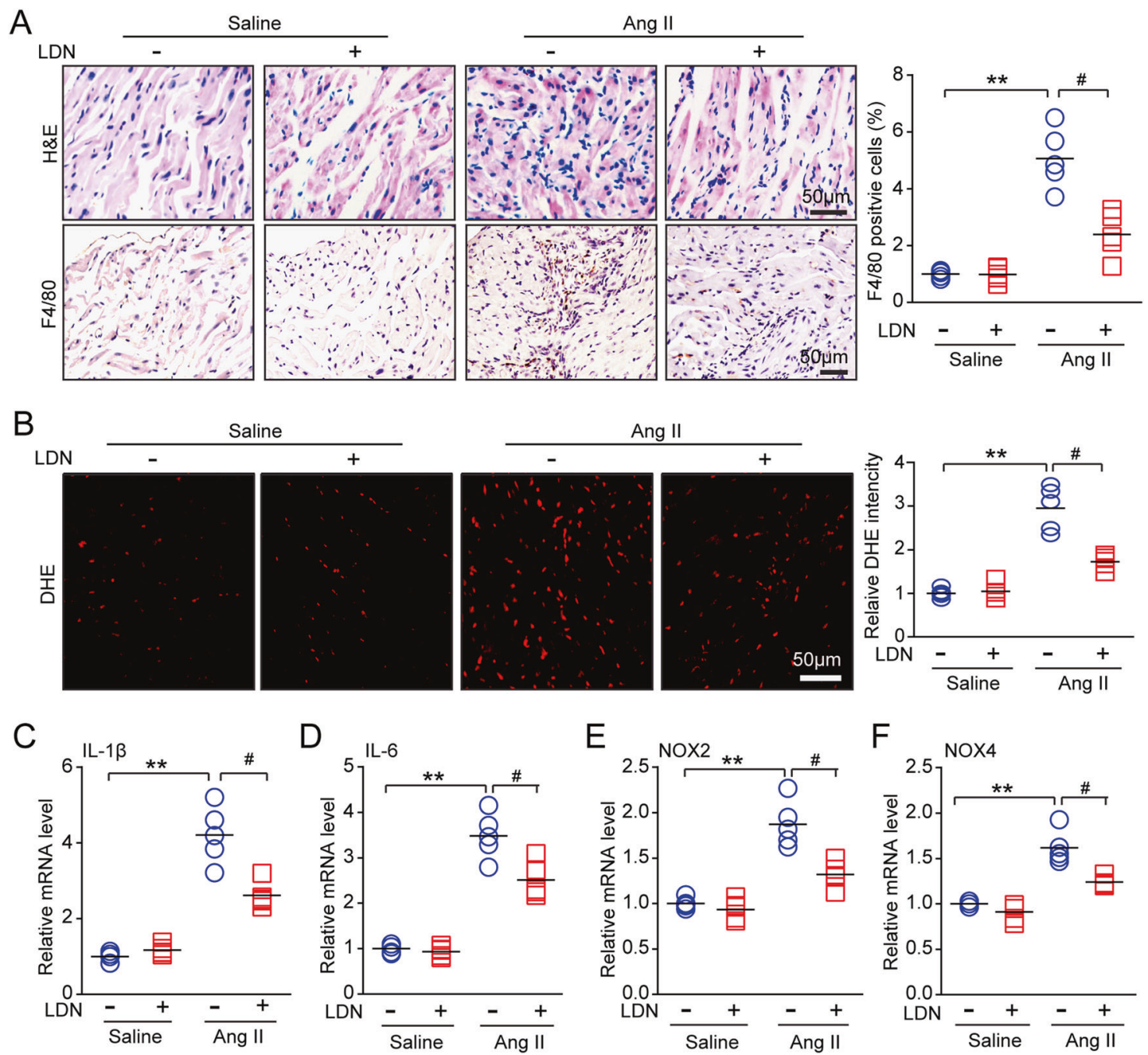

Fig. 5 Administration of LDN reduces Ang II-induced atrial inflammation. a Representative hematoxylin-eosin (H\&E) staining (top) and F4/80 (macrophage marker) immunohistochemistry (bottom) in the atrial tissues of DMSO-treated and LDN-treated mice after infusion with saline or Ang II for $21 \mathrm{~d}$ ( $n=5$ mice per group). The quantification of F4/80-positive cells (right; $n=5$ mice per group). b Dihydroethidium (DHE) staining of atrial tissues from DMSO-treated and

LDN-treated mice after infusion with saline or Ang II for $21 \mathrm{~d}$ (left). The quantification of fold changes in DHE intensity (right; $n=5$ mice per group). c-f qPCR analyses of proinflammatory cytokine (IL-1 $\beta$ and IL-6) and NADPH oxidase subunit (NOX2 and NOX4) levels in atrial tissues $(n=5) . \mathrm{n}$ represents the number of animals. ${ }^{*} P<0.05$, ${ }^{* *} P<0.01$ ' or ${ }^{\#} P<0.05$ versus saline-treated WT mice or Ang IItreated WT mice

induces CX43 expression via the Smad and ERK1/2 signaling pathways [30], which are involved in Ang IIstimulated AF [28]. Interestingly, these proinflammatory and profibrotic pathways are regulated by AKT, which activates NF-kB, NOX, and TGF- $\beta /$ Smad signaling $[20,31]$. Notably, recent data have demonstrated that UCHL1 can interact with AKT and lead to the activation of AKT in MCF-7 cells [32]. Whether the inhibition of UCHL1 blocks AKT and multiple downstream mediators remains unclear. LDN is a potent reversible, competitive and active site-directed inhibitor of UCHL1. The administration of LDN aggravates mossy fiber sprouting and induces LC3 puncta formation and autophagy in the HeLa cell line and apoptosis through the activation of endoplasmic reticulum stress (ERS) [33, 34]. Here, our results demonstrated that the treatment of mice with LDN significantly inhibited the Ang II-induced activation of AKT, ERK1/2, HIF-1 $\alpha$, TGF- $\beta / \mathrm{Smad} 2 / 3$, IL-1, IL-6, and NOX, thereby leading to the improvement of atrial remodeling and AF (Figs. 4-6). Thus, the results provide novel insights into the mechanisms of Ang II-induced AF as well as into the protective effect of UCHL1 against AF. This effect is consistent with previous findings that LDN inhibits the activation of ERK and AKT in human neuroblastoma cell lines [35], suggesting that UCHL1 regulates these signaling pathways in different cell types.

Notably, although the reduction in blood pressure induced by LDN in Ang II-infused mice was significant, the 
Fig. 6 Treatment of LDN suppresses Ang II-induced oxidative stress, inflammation and downstream mediators in mice. a Immunoblotting analyses of the profibrotic signaling molecules p-AKT, AKT, pERK1/2, ERK1/2, and tubulin in the heart. The quantification of the relative protein levels $(n=4)$. b Immunoblotting analyses of fibrosis, oxidative stress and the inflammatory mediators HIF- $1 \alpha$, TGF- $\beta 1$, p-Smad2/3, Smad2,

Smad3, and CX43 in the heart. The quantification of the relative protein levels $(n=4)$. $\alpha$-Tubulin was used as an internal control. $n$ represents the number of animals $(n=4) .{ }^{*} P<0.05,{ }^{* *} P<0.01$ versus saline control; ${ }^{\#} P<0.05$ versus Ang II alone. c A working model of the effect of LDN administration on Ang II-induced AF. Treatment with LDN suppresses Ang II-induced oxidative stress and inflammatory mediators (AKT, ERK1/2, HIF-1 $\alpha$, and TGF- $\beta /$ Smad2/3) in mice
A

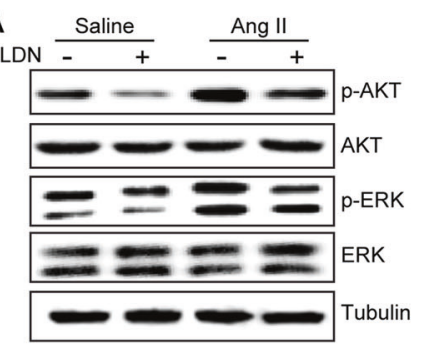

B

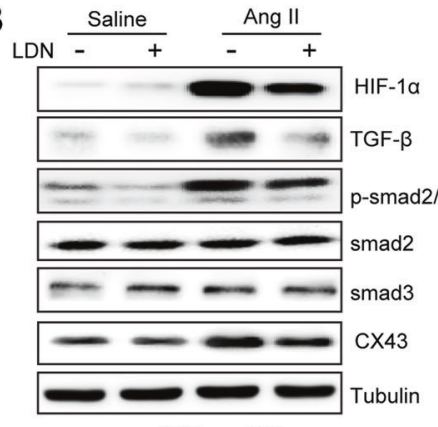

$\mathrm{p}-\mathrm{smad} 2 / 3 / \mathrm{smad} 2 / 3$
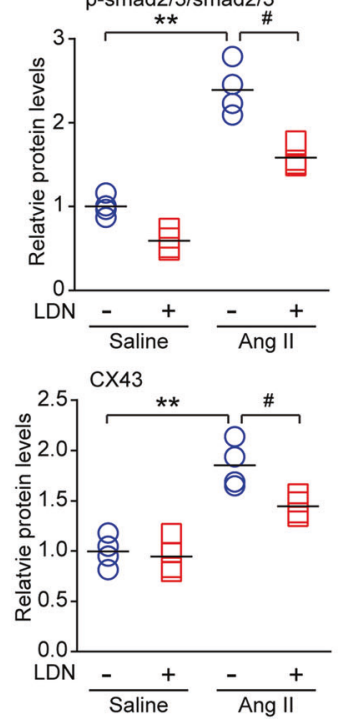
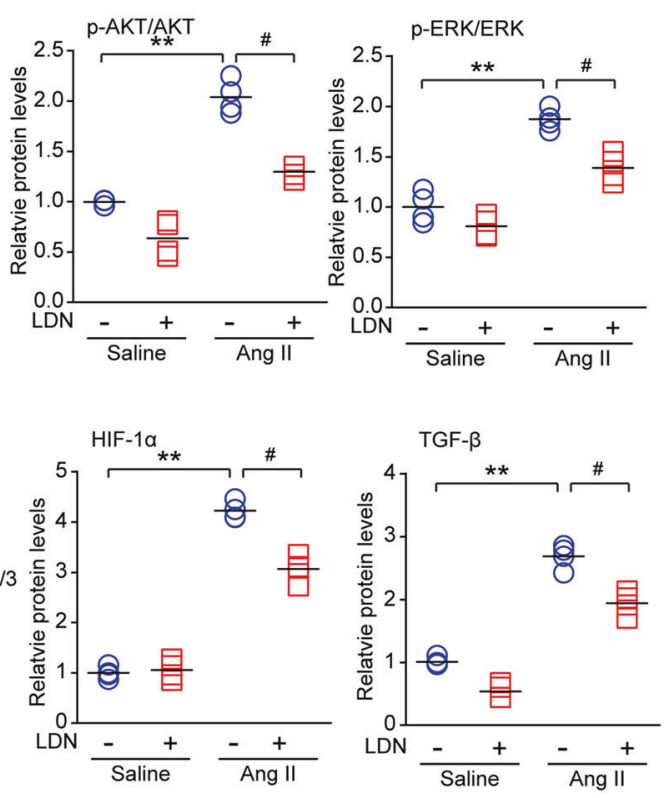

C
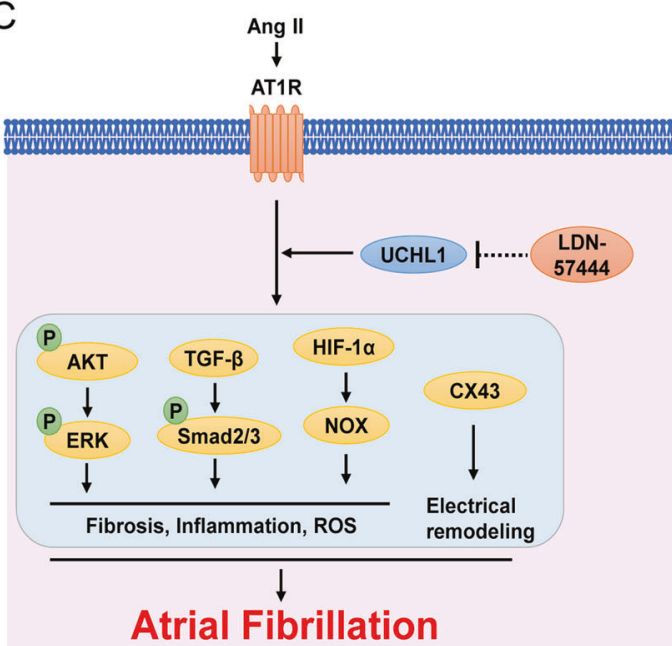

Atria
SBP was still higher than that in saline-infused controls (Fig. 2a), suggesting that the administration of LDN partially reduced hypertension. Furthermore, LDN markedly attenuated Ang II-induced left atrial remodeling, inflammation and oxidative stress as well as the activation of multiple signals (AKT, ERK1/2, HIF- $1 \alpha$, TGF- $\beta / \operatorname{smad} 2 / 3$, and CX43) in the atria, suggesting that LDN inhibited AF possibly through blood pressure-dependent and blood pressure-independent manners. How hypertension is involved in the LDN-mediated beneficial effect against AF and whether low-dose LDN has the same effect on AF need to be tested in a pressure overload-induced AF model in the future.

In conclusion, this study clarifies a new role for the UCHL1 inhibitor LDN in Ang II-induced AF and the possible mechanism. These data suggest that LDN may have potential clinical applications for the treatment of hypertensive AF and related heart diseases. In the future, it is necessary to further investigate how hypertrophic stimulation upregulates the expression of UCHL1 in atrial tissues and to assess the role of LDN in other animal models.

Acknowledgements This work was supported by grants from the National Natural Science Foundation of China $(81700350,81330003$, 31571170, and 81630009) and Dalian High Level Talents Innovation and Entrepreneurship Projects (2015R019).

Authors contributions HHL and HLB conceived and designed the experiments. HLB, YLZ, JY, QS, XLY, XY, CC, and ZL performed the experiments. HLB, YLZ, JY, QS, XLY, and XY analyzed the 
experimental data. HHL and HLB wrote the manuscript. All authors read and approved the manuscript.

\section{Compliance with ethical standards}

Conflict of interest The authors declare that they have no conflict of interest.

Publisher's note Springer Nature remains neutral with regard to jurisdictional claims in published maps and institutional affiliations.

Open Access This article is licensed under a Creative Commons Attribution 4.0 International License, which permits use, sharing, adaptation, distribution and reproduction in any medium or format, as long as you give appropriate credit to the original author(s) and the source, provide a link to the Creative Commons license, and indicate if changes were made. The images or other third party material in this article are included in the article's Creative Commons license, unless indicated otherwise in a credit line to the material. If material is not included in the article's Creative Commons license and your intended use is not permitted by statutory regulation or exceeds the permitted use, you will need to obtain permission directly from the copyright holder. To view a copy of this license, visit http://creativecommons. org/licenses/by/4.0/.

\section{References}

1. Singh B. Atrial fibrillation: from ion channels to bedside treatment options. J Electrocardiol. 2009;42:660-70.

2. Kimura S, Ito M, Tomita M, Hoyano M, Obata H, Ding L, et al. Role of mineralocorticoid receptor on atrial structural remodeling and inducibility of atrial fibrillation in hypertensive rats. Hypertens Res. 2011;34:584-91.

3. Tan AY, Zimetbaum P. Atrial fibrillation and atrial fibrosis. J Cardiovasc Pharm. 2011;57:625-9.

4. Li J, Wang S, Bai J, Yang XL, Zhang YL, Che YL, et al. Novel role for the immunoproteasome subunit PSMB10 in angiotensin II-induced atrial fibrillation in mice. Hypertension. 2018;71: 866-76.

5. Bunch TJ, Mahapatra S, Bruce GK, Johnson SB, Miller DV, Horne $\mathrm{BD}$, et al. Impact of transforming growth factor-betal on atrioventricular node conduction modification by injected autologous fibroblasts in the canine heart. Circulation. 2006;113: 2485-94.

6. Spronk HM, De Jong AM, Verheule S, De Boer HC, Maass AH, Lau DH, et al. Hypercoagulability causes atrial fibrosis and promotes atrial fibrillation. Eur Heart J. 2017;38:38-50.

7. Gramley F, Lorenzen J, Jedamzik B, Gatter K, Koellensperger E, Munzel $\mathrm{T}$, et al. Atrial fibrillation is associated with cardiac hypoxia. Cardiovasc Pathol. 2010;19:102-11.

8. Pan CH, Lin JL, Lai LP, Chen CL, Stephen Huang SK, Lin CS. Downregulation of angiotensin converting enzyme II is associated with pacing-induced sustained atrial fibrillation. FEBS Lett. 2007;581:526-34.

9. Tsai CT, Lai LP, Hwang JJ, Chen WP, Chiang FT, Hsu KL, et al. Renin-angiotensin system component expression in the HL-1 atrial cell line and in a pig model of atrial fibrillation. J Hypertens. 2008;26:570-82.

10. Kulathu Y, Komander D. Atypical ubiquitylation - the unexplored world of polyubiquitin beyond Lys48 and Lys63 linkages. Nat Rev Mol Cell Biol. 2012;13:508-23.

11. Liu Y, Fallon L, Lashuel HA, Liu Z, Lansbury PT Jr. The UCHL1 gene encodes two opposing enzymatic activities that affect alpha-synuclein degradation and Parkinson's disease susceptibility. Cell. 2002;111:209-18.

12. Choi J, Levey AI, Weintraub ST, Rees HD, Gearing M, Chin LS, et al. Oxidative modifications and down-regulation of ubiquitin carboxyl-terminal hydrolase L1 associated with idiopathic Parkinson's and Alzheimer's diseases. J Biol Chem. 2004;279: 13256-64.

13. Rani A, Roy S, Singh M, Devi U, Yadav RK, Gautam S, et al. alpha-Chymotrypsin regulates free fatty acids and UCHL-1 to ameliorate N-methyl nitrosourea induced mammary gland carcinoma in albino wistar rats. Inflammopharmacology. 2016;24: 277-86.

14. Wilson CL, Murphy LB, Leslie J, Kendrick S, French J, Fox CR, et al. Ubiquitin C-terminal hydrolase 1: A novel functional marker for liver myofibroblasts and a therapeutic target in chronic liver disease. J Hepatol. 2015;63:1421-8.

15. Yamazaki K, Wakasugi N, Tomita T, Kikuchi T, Mukoyama M, Ando K. Gracile axonal dystrophy (GAD), a new neurological mutant in the mouse. Proc Soc Exp Biol Med. 1988;187:209-15.

16. Drobysheva A, Ahmad M, White R, Wang HW, Leenen FH. Cardiac sympathetic innervation and PGP9.5 expression by cardiomyocytes after myocardial infarction: effects of central MR blockade. Am J Physiol Heart Circ Physiol. 2013;305:H1817-29.

17. Purohit A, Rokita AG, Guan X, Chen B, Koval OM, Voigt N, et al. Oxidized $\mathrm{Ca}(2+) /$ calmodulin-dependent protein kinase II triggers atrial fibrillation. Circulation. 2013;128:1748-57.

18. Wang L, Zhang YL, Lin QY, Liu Y, Guan XM, Ma XL, et al. CXCL1-CXCR2 axis mediates angiotensin II-induced cardiac hypertrophy and remodelling through regulation of monocyte infiltration. Eur Heart J. 2018;39:1818-31.

19. Wang L, Zhao XC, Cui W, Ma YQ, Ren HL, Zhou X, et al. Genetic and pharmacologic inhibition of the chemokine receptor CXCR2 prevents experimental hypertension and vascular dysfunction. Circulation. 2016;134:1353-68.

20. Li J, Wang S, Zhang YL, Bai J, Lin QY, Liu RS, et al. Immunoproteasome subunit beta5i promotes Ang II (Angiotensin II)induced atrial fibrillation by targeting ATRAP (Ang II type I receptor-associated protein) degradation in mice. Hypertension. 2019;73:92-101.

21. Korantzopoulos P, Letsas KP, Tse G, Fragakis N, Goudis CA, Liu T. Inflammation and atrial fibrillation: a comprehensive review. $\mathrm{J}$ Arrhythm. 2018;34:394-401.

22. Liu C, Zhang JW, Hu L, Song YC, Zhou L, Fan Y, et al. Activation of the AT1R/HIF-1 alpha /ACE axis mediates angiotensin II-induced VEGF synthesis in mesenchymal stem cells. Biomed Res Int. 2014;2014:627380.

23. Fang Y, Shen X. Ubiquitin carboxyl-terminal hydrolases: involvement in cancer progression and clinical implications. Cancer Metastasis Rev. 2017;36:669-82.

24. Gao H, Hartnett S, Li Y. Ubiquitin C-Terminal Hydrolase L1 regulates myoblast proliferation and differentiation. Biochem Biophys Res Commun. 2017;492:96-102.

25. Rajagopalan V, Zhao M, Reddy S, Fajardo G, Wang X, Dewey S, et al. Altered ubiquitin-proteasome signaling in right ventricular hypertrophy and failure. Am J Physiol Heart Circ Physiol. 2013; 305:H551-562.

26. Chu KY, Li H, Wada K, Johnson JD. Ubiquitin C-terminal hydrolase L1 is required for pancreatic beta cell survival and function in lipotoxic conditions. Diabetologia. 2012;55:128-40.

27. Costes S, Gurlo T, Rivera JF, Butler PC. UCHL1 deficiency exacerbates human islet amyloid polypeptide toxicity in beta-cells: evidence of interplay between the ubiquitin/proteasome system and autophagy. Autophagy. 2014;10:1004-14.

28. Gao G, Dudley SC Jr. Redox regulation, NF-kappaB, and atrial fibrillation. Antioxid Redox Signal. 2009;11:2265-77. 
29. Semenza GL. HIF-1, O(2), and the 3 PHDs: how animal cells signal hypoxia to the nucleus. Cell. 2001;107:1-3.

30. Cheng JC, Chang HM, Fang L, Sun YP, Leung PC. TGF-beta1 upregulates connexin43 expression: a potential mechanism for human trophoblast cell differentiation. J Cell Physiol. 2015;230:1558-66.

31. Li J, Liu X, Zheng S, Huang Y. Use of oral anticoagulation for patients with atrial fibrillation and end-stage renal disease: what is needed nowadays? Rev Esp Cardiol. 2019;72:96-97.

32. Luo Y, He J, Yang C, Orange M, Ren X, Blair N, et al. UCH-L1 promotes invasion of breast cancer cells through activating Akt signaling pathway. J Cell Biochem. 2018;119:691-700.
33. Wen Y, Wu Q, Shi Q, Xie Y, Dan W, Chen Y, et al. UCH-L1 inhibition aggravates mossy fiber sprouting in the pentylenetetrazole kindling model. Biochem Biophys Res Commun. 2018;503:2312-8.

34. Yan C, Huo H, Yang C, Zhang T, Chu Y, Liu Y. Ubiquitin Cterminal hydrolase L1 regulates autophagy by inhibiting autophagosome formation through its deubiquitinating enzyme activity. Biochem Biophys Res Commun. 2018;497:726-33.

35. Gu Y, Lv F, Xue M, Chen K, Cheng C, Ding X, et al. The deubiquitinating enzyme UCHL1 is a favorable prognostic marker in neuroblastoma as it promotes neuronal differentiation. J Exp Clin Cancer Res. 2018;37:258. 